\title{
Photoconductivity Multiplication in Semiconducting Few-Layer $\mathrm{MoTe}_{2}$
}

\author{
Wenhao Zheng, Mischa Bonn, and Hai I. Wang*
}

Cite This: Nano Lett. 2020, 20, 5807-5813

Read Online

ABSTRACT: We report efficient photoconductivity multiplication in few-layer $2 \mathrm{H}$ $\mathrm{MoTe}_{2}$ as a direct consequence of an efficient steplike carrier multiplication with near unity quantum yield and high carrier mobility $\left(\sim 45 \mathrm{~cm}^{2} \mathrm{~V}^{-1} \mathrm{~s}^{-1}\right)$ in $\mathrm{MoTe}_{2}$. This photoconductivity multiplication is quantified using ultrafast, excitation-wavelengthdependent photoconductivity measurements employing contact-free terahertz spectroscopy. We discuss the possible origins of efficient carrier multiplication in $\mathrm{MoTe}_{2}$ to guide future theoretical investigations. The combination of photoconductivity multiplication and the advantageous bandgap renders $\mathrm{MoTe}_{2}$ as a promising candidate for efficient optoelectronic devices.

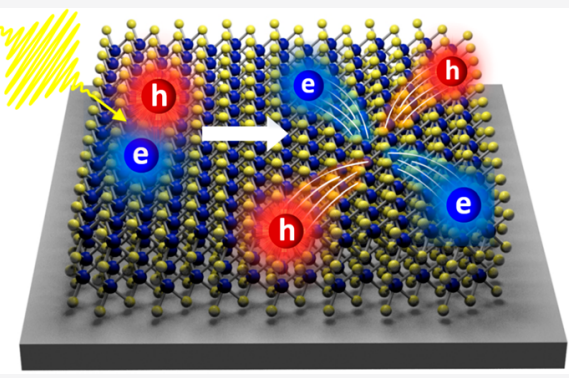

KEYWORDS: carrier multiplication, impact ionization, transition metal dichalcogenides (TMDCs), 2D materials, MoTe, terahertz spectroscopy

\section{INTRODUCTION}

Photoexcitation of semiconductors with photon energies $(h v)$ exceeding their bandgap $\left(E_{\mathrm{g}}\right)$ creates energetic electrons and holes. In most semiconductors, these so-called hot carriers can cool down to the band-edge via carrier-phonon scattering, on a subpicosecond time scale. Such ultrafast energy loss via hot carrier cooling accounts for over $\sim 30 \%$ efficiency reduction within the Shockley and Queisser framework. ${ }^{1}$

Optical generation of multiple pairs of electrons and holes in semiconductors by an energetic photon (with $h v>2 E_{\mathrm{g}}$ ), a process known as carrier multiplication (CM) or multiexciton generation (MEG), ${ }^{2}$ provides a novel solution to circumvent the energy losses in hot carriers relevant for high-efficiency photovoltaics. Substantial previous research has aimed at quantifying the $\mathrm{CM}$ efficiency and obtaining a fundamental understanding of the $\mathrm{CM}$ mechanism with a primary focus on semiconducting quantum dots (QDs). ${ }^{3-11}$ The benefits from strong quantum confinement for CM in QDs come from the enhanced Coulomb interaction and relaxed momentum conservation condition. On the other hand, previous theoretical $^{12,13}$ and experimental ${ }^{14-17}$ studies have also shown that the reduced density of states imposed by confinement may constrain the energy conservation condition so that quantum confinement does not necessarily facilitate CM.

In this context, two-dimensional materials with atomic-level control of the thickness, combined with strong Coulomb effects $^{18,19}$ and still relatively high density of states, are potentially interesting platforms for efficient CM. Indeed, Aerts et al. reported a strong MEG effect in thin $\mathrm{PbS}$ nanosheets with much higher efficiency than that in zero-dimensional
QDs, one-dimensional nanorods, and 3D bulk counterparts. ${ }^{20}$ Furthermore, Kim and colleagues extended recently such studies into multilayered van der Waals (vdW) materials of $\mathrm{MoTe}_{2}$ and $\mathrm{WSe}_{2}{ }^{21}{ }^{21}$ They reported near-perfect MEG in vdW structures employing transient absorption (TA) spectroscopy, that is, a unity quantum yield with an onset energy of $2 E_{\mathrm{g}}$. However, the mechanism on which the CM/MEG takes place in vdW materials remains elusive. While the generation of multiexcitons following MEG was proposed, ${ }^{21}$ direct spectroscopic evidence for these high-order excitonic states is missing. Finally, if indeed (multi)excitons are the primary photoproduct, they would have to be dissociated, for example, for photovoltaic applications. Therefore, establishing the nature of the multiple carrier generation process by $\mathrm{CM} / \mathrm{MEG}$ in vdW structures is relevant for applications.

\section{RESULTS AND DISCUSSION}

Structural Characterization. Figure 1a shows the atomic configuration of the $2 \mathrm{H}$ phase of $\mathrm{MoTe}_{2}$. The sample is produced by chemical vapor deposition (from SixCarbon Technology, Shenzhen), and the layer thickness is about $5 \mathrm{~nm}$ $(=\sim 7$ layers $)$. The Raman spectrum in Figure $1 \mathrm{~b}$ exhibits two prominent peaks at 173 and $235 \mathrm{~cm}^{-1}$ originating from the out-of-plane $A_{1 \mathrm{~g}}$ mode and in-plane $E_{2 \mathrm{~g}}$ mode in $2 \mathrm{H}$ -

Received: April 20, 2020

Revised: July 22, 2020

Published: July 22, 2020 
(a)

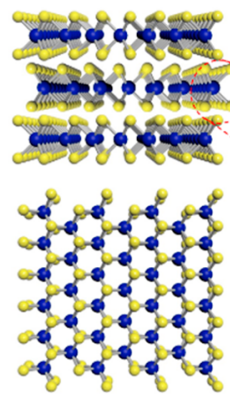

(b)

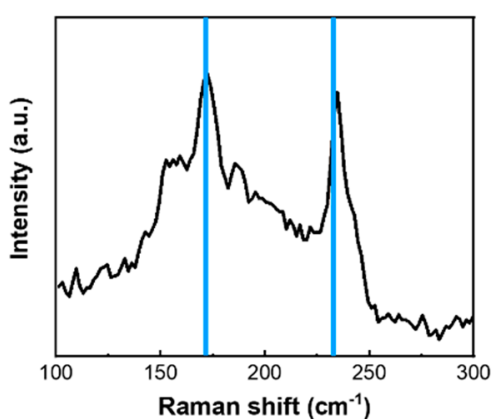

(c)

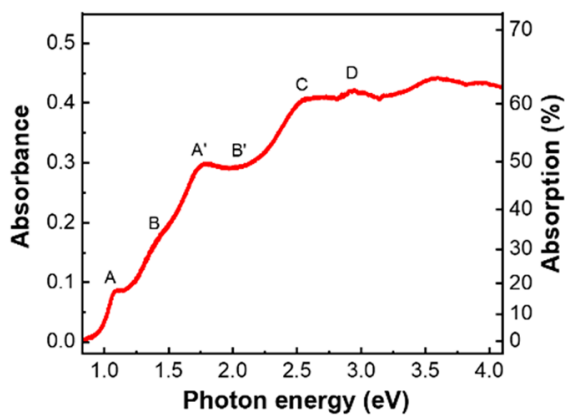

Figure 1. Characterization of the few-layer $2 \mathrm{H}-\mathrm{MoTe}_{2}$ sample. (a) The schematic diagrams of $2 \mathrm{H}$ phase structure of $\mathrm{MoTe}_{2}$. (b) Raman spectrum of the $2 \mathrm{H}-\mathrm{MoTe}_{2}$ with marks from the computational results. ${ }^{24}$ (c) UV-vis absorption spectrum of $2 \mathrm{H}-\mathrm{MoTe}_{2}$.

(a)

(b)

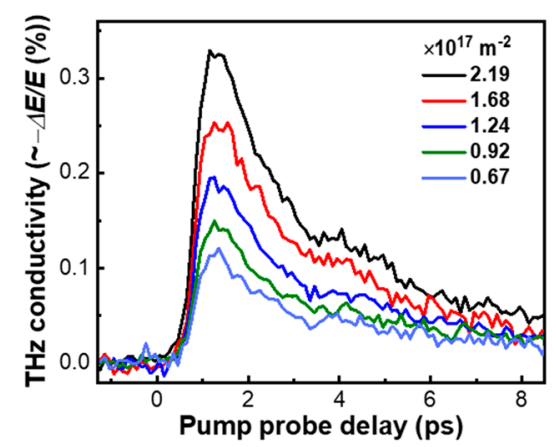

(d)

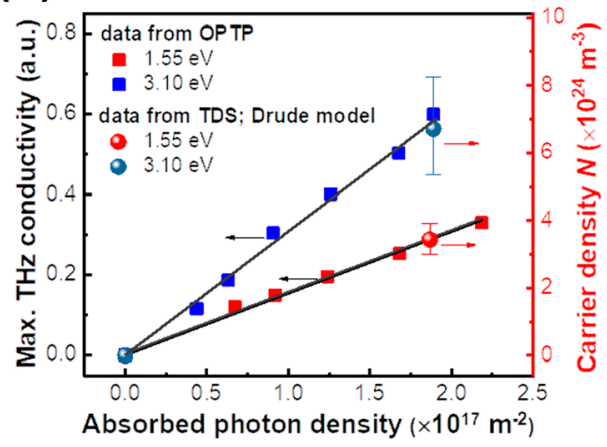

(c)

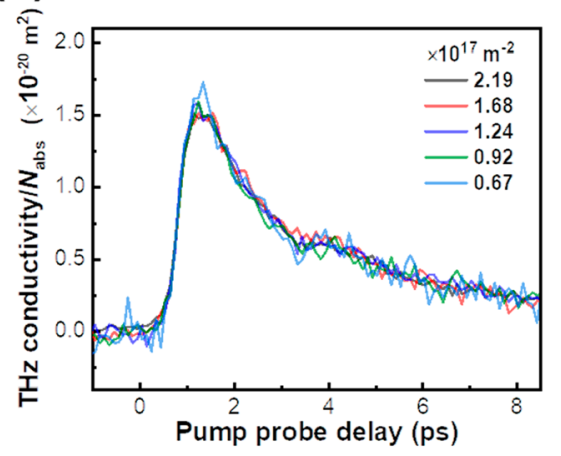

(e)

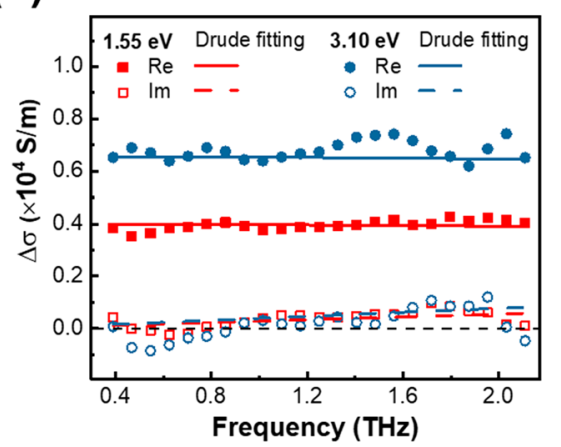

Figure 2. Investigation of $\mathrm{CM}$ in $\mathrm{MoTe}_{2}$ by THz spectroscopy. (a) Schematic illustration of the optical-pump THz-probe spectroscopy. (b) The fluence-dependent, time-resolved $\mathrm{THz}$ conductivity following $1.55 \mathrm{eV}$ excitation. The bump at 4 ps originates from re-excitation of the sample by the reflected pulse from the substrate/air interface. (c) The THz conductivities normalized to the absorbed photon density based on the data in (b). (d) Illustration of CM effect by the fluence-dependent THz photoconductivity peak values (left $y$-axis) and rescaled photogenerated carrier density inferred from the fitting in (e). (e) Frequency-resolved $\mathrm{THz}$ conductivity measured with Drude fitting, following excitations of 1.55 and $3.10 \mathrm{eV}$. 
$\mathrm{MoTe}_{2}{ }^{22-24}$ Optically, multilayered $\mathrm{MoTe}_{2}$ has been shown to possess an indirect bandgap. ${ }^{25}$ To quantify its bandgap $E_{\mathrm{g}}$, we have conducted UV-vis absorption measurements. On the basis of the absorbance in Figure 1c, we quantify the bandgap of $2 \mathrm{H}-\mathrm{MoTe}_{2}$ to be $0.90 \mathrm{eV}$ using a Tauc plots (see Figure S1). Other optical transitions, marked by $\mathrm{A}, \mathrm{B}, \mathrm{A}^{\prime}, \mathrm{B}^{\prime}, \mathrm{C}$, and $\mathrm{D}$, correspond to direct transitions at the $K$ and $\Gamma$ points of the Brillouin zone. ${ }^{26}$ Note that the absorption of multilayered $\mathrm{MoTe}_{2}$ on sapphire does not show the absorption quanta of $\sim 1.6 \%$ observed by Fang et al. for InAs thin films, presumably due to the indirect nature of transitions in $\mathrm{MoTe}_{2} .{ }^{27}$ For monolayer $\mathrm{MoTe}_{2}$, strong exciton effects have been reported with binding energy up to $600 \mathrm{meV} \cdot{ }^{28-30}$ For the bulk phase, the exciton binding energy is small at $\sim 15 \mathrm{meV}$, substantially lower than the thermal excitation at room temperature $\left(k_{\mathrm{B}} T\right) .^{31}$

Carrier Multiplication in $2 \mathrm{H}-\mathrm{MoTe}_{2}$. To explore the CM effect, we employ optical-pump terahertz ( $\mathrm{THz}$ )-probe (OPTP) spectroscopy to measure the ultrafast photoconductivity. As a purely optical technique, OPTP spectroscopy has been demonstrated to provide quantitative insight into the intrinsic electrical transport properties (including charge density, scattering time, and so forth) in a contact-free manner. ${ }^{32-35}$ In a typical experiment, as shown in Figure 2a, a laser pulse with tunable photon energies above the bandgap is used to excite electrons from valence to conduction band. Subsequently, a single-cycle $\mathrm{THz}$ pulse with $\sim 1$ ps duration is employed to probe the time-dependent photoconductivity $(\Delta \sigma)$ dynamics by monitoring the photoinduced $\mathrm{THz}$ absorption $(\Delta E)$ at varied pump-probe delays. The photoconductivity is proportional to $\Delta E$ by $\Delta \sigma=-\Delta E=-\left(E_{\text {pump }}-\right.$ $\left.E_{0}\right)$, where $E_{\text {pump }}$ and $E_{0}$ represent the transmitted $\mathrm{THz}$ field with and without photoexcitation.

Figure $2 \mathrm{~b}$ shows the fluence-dependent $\mathrm{THz}$ photoconductivity following $1.55 \mathrm{eV}$ excitation. This transient photoconductivity exhibits a subpicosecond rise, reflecting free carrier generation, and a fast decay within a few picoseconds. We attribute this fast decay to ultrafast carrier trapping at defects (see the extended discussion in SI) in line with previous studies. ${ }^{21,36}$ While the passivation of these defects will be required for device applications, it does not affect the CM process in this study as CM occurs on a subpicosecond time scale. ${ }^{21}$ In Figure $2 c$, we present the photoconductivities normalized to the absorbed photon density. We find that within the fluence ranges used in this study, the photoconductivities scale linearly with fluence. On the basis of these results, we can exclude the multiplication of charge carriers trivially by a two-photon excitation process.

To investigate the $\mathrm{CM}$ in $\mathrm{MoTe}_{2}$, we have further measured pump photon energy-dependent photoconductivities. The conductivity dynamics following the $3.1 \mathrm{eV}$ excitation is shown in Figure S2a. In Figure 2d, we compare the fluencedependent peak value in photoconductivity of $\mathrm{MoTe}_{2}$ under 3.1 and $1.55 \mathrm{eV}$ excitations, corresponding to $\sim 3.4$ ( $\mathrm{CM}$ energetically possible) and 1.7 (CM impossible) times of the bandgap, respectively. For a given absorbed photon density, we find that the photoconductivity upon $3.1 \mathrm{eV}$ excitation is nearly twice that of $1.55 \mathrm{eV}$ excitation. While this photoconductivity multiplication provides a strong indication of possible CM effect in $\mathrm{MoTe}_{2}$, special care needs to be taken for data analysis. Particularly, the photoconductivity $\Delta \sigma$ scales with the products of carrier density $(N)$ and charge mobility $(\mu)$ as $\Delta \sigma$ $=N \cdot e \cdot \mu$ with $e$ as the elementary charge. To separate the contribution of the carrier density from the charge mobility, we have conducted $\mathrm{THz}$ time-domain spectroscopy (TDS, see SI) at the pump-probe delay close to the photoconductivity peak (at a pump-probe delay of $1.5 \mathrm{ps}$ ).

In Figure 2e, we compare the frequency-resolved photoconductivities for both 3.1 and $1.55 \mathrm{eV}$ excitations obtained from TDS measurements. In both cases, the conductivities display a large real conductivity and essentially zero imaginary contribution. This result suggests that a substantial amount (if not all) of photogenerated carriers in our sample are present as free charges. Importantly, the free carrier response dominates the dynamics for all excitations so that we can rule out the free charge generation via "hot exciton dissociation", one of the most reported free charge generation mechanisms in excitonic materials. ${ }^{37,38}$

To further shed light on the electrical transport properties and disentangle the contribution of carrier density from mobility in $\mathrm{MoTe}_{2}$ at a given pump-probe delay time, we apply the standard Drude model (see SI). ${ }^{32}$ We find that the model describes the data well, and we summarize the inferred parameters in Table 1. When comparing 3.1 and $1.55 \mathrm{eV}$

Table 1. Fitting Parameters for Electrical Charge Transport Properties Based on the Drude Model

\begin{tabular}{ccc} 
& \multicolumn{2}{c}{ Drude model } \\
\cline { 2 - 3 } excitation & $\omega_{P}(\mathrm{THz})$ & $\tau(\mathrm{fs})$ \\
$1.55 \mathrm{eV}$ & $200 \pm 13$ & $11 \pm 2$ \\
$3.10 \mathrm{eV}$ & $279 \pm 28$ & $10 \pm 2$ \\
\hline
\end{tabular}

excitations, the charge scattering times $\tau$ are found to be nearly identical; the only different parameter is the plasma frequency $\omega_{p}$, which is directly related to carrier density N. Microscopically, the charge carrier mobility $\mu$ in a material is given by $\mu=\frac{\mathrm{e} \cdot \tau}{m^{*}}$. The same $\tau$ for both 3.1 and $1.55 \mathrm{eV}$ excitations implies that charge carrier mobility does not depend on the excitation photon energies at the time delay chosen for TDS (1.5 ps after photoexcitation). To make a quantitative correlation, we have plotted the carrier densities obtained from the fittings as the right $Y$-axis and compared them to the fluence-dependent photoconductivity data, as shown in Figure $2 \mathrm{~d}$. The overlap between rescaled carrier densities and photoconductivities offers direct, quantitative evidence for efficient CM taking place in our sample. Note that the conclusion is robust and does not depend on the model applied. Strong support for this argument comes from the normalized frequency-resolved conductivity, as shown in Figure S4 (see SI) based on the same data sets in Figure 2e, and we find no observable difference between the two. An extended analysis based on the Drude-Smith model leads to the same conclusion of CM (see Figure S5 and associated discussion in SI). This result also indicates that charge carriers reach a quasi-equilibrium condition (e.g., at the band-edge) at 1.5 ps after the excitation with both 1.5 (via cooling or phonon emission processes) and $3.1 \mathrm{eV}$ (by CM) photons.

Finally, based on the $\tau$ inferred from the fittings, we calculate the $d c$ electron mobility in our sample to be $45 \pm 9 \mathrm{~cm}^{2} \mathrm{~V}^{-1}$ $\mathrm{s}^{-1}$. The value is much higher than that of the QD solids (with the reported value in most studies way below $1 \mathrm{~cm}^{2} \mathrm{~V}^{-1} \mathrm{~s}^{-1}$ and the highest around $\left.10 \mathrm{~cm}^{2} \mathrm{~V}^{-1} \mathrm{~s}^{-1}\right) \cdot{ }^{39,40}$ In addition, the estimated mobility is in the same range as the results $(\sim 30-40$ $\mathrm{cm}^{2} \mathrm{~V}^{-1} \mathrm{~s}^{-1}$ ) from electrical transport measurements on 
(a)

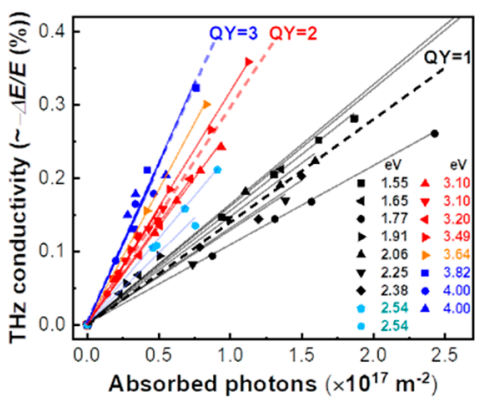

(b)

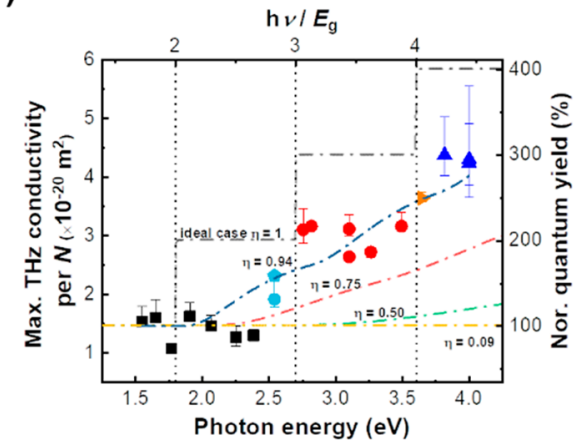

(c)

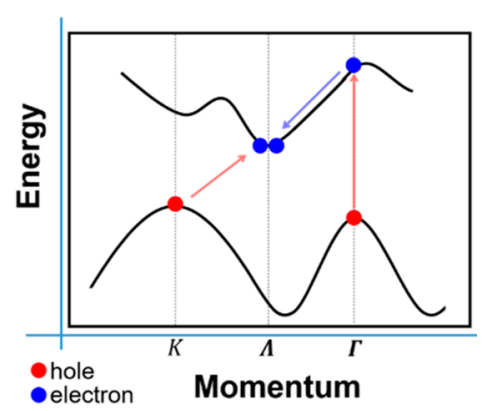

Figure 3. Determination of the carrier-multiplication efficiency. (a) The peak value of $\mathrm{THz}$ photoconductivity of $2 \mathrm{H}-\mathrm{MoTe}_{2}$ as a function of absorbed photon density for different pump photon energies. The solid lines are linear fittings to the data; three dashed lines represent CM quantum yield of $100 \%, 200 \%$, and $300 \%$, to guide the eye. (b) The photoconductivity at a given absorbed photon density for varied photon energies ( $h \nu$, bottom $x$-axis) and the ratio of $h \nu / E_{\mathrm{g}}$ (top $x$-axis). The data are rescaled to match $100 \%$ quantum yield of charge generation for the sub-2 $E_{\mathrm{g}}$ excitations. The model (dash-dotted lines) is discussed in the main text. (c) Schematic drawing of band structure and carrier multiplication in indirect semiconducting few-layer $\mathrm{MoTe}_{2}$.

$\mathrm{MoTe}_{2}$ thin films. ${ }^{41,42}$ As such, we conclude that $2 \mathrm{D} \mathrm{MoTe}_{2}$ multilayers uniquely combine efficient $\mathrm{CM}$ with high carrier conductivity.

Quantification of CM Efficiency in $\mathrm{MoTe}_{2}$. To evaluate the CM quantum yields, we performed the pump wavelengthdependent OPTP measurements on $2 \mathrm{H}-\mathrm{MoTe}_{2}$ with up to 16 different pump photon energies. The conductivity maximum versus fluence is shown in Figure 3a. Here, we take the photoconductivity maximum at a given absorbed photon density (or equivalently the slope in the fluence-dependent conductivity in Figure 3a) to quantify the $\mathrm{CM}$ efficiency, as these two values are linked in a linear fashion (see Figure $2 \mathrm{~d}$ ). In Figure 3b, we summarize the photoconductivity normalized to the absorbed photon density at varied photon energies. For the range of excitation energies between 1 and 2 times that of $E_{\mathrm{g}}$ (no $\mathrm{CM}$ ), we find that the carrier generation efficiency is independent of the pump photon energies, as expected. For further data analysis, we assume the carrier generation quantum yield for photon energy below twice the $E_{\mathrm{g}}$ to be $100 \%$ (marked in the right $Y$-axis). With increasing the photon energy, we observe twice photoconductivity jumps at around 2.8 and 4.2 times of $E_{\mathrm{g}}$, which we attribute to CM effect following the previous discussion. Remarkably, we observe the enhancement of photoconductivity by $\mathrm{CM}$ by a steplike manner with nearly $100 \% \mathrm{CM}$ quantum yields. ${ }^{43}$ Finally, we investigate the recombination dynamics following CM. In QD systems, multiple pairs of electrons and holes generated by $\mathrm{CM}$ can effectively recombine in a few to tens of picoseconds via Auger recombination. ${ }^{2,8,10,44}$ In contrast, as shown in Figure S6, for all pump energies used in the study, we observe no changes in the decay dynamics. Apparently, the recombination process is dominated by the fast trapping by defects, which effectively competes with the Auger process. Note that this was previously also concluded by Kim et al. ${ }^{21}$

Discussion. Now we discuss the mechanism by which CM in multilayer $\mathrm{MoTe}_{2}$ takes place. The free carrier dominant dynamics observed in all pump photon energies implies that $\mathrm{CM}$ in our system occurs through a scattering mechanism known as impact ionization (II). ${ }^{12,45-48}$ In the II process, a hot carrier with excess energy beyond the bandgap can effectively re-excite an additional electron across the bandgap on subpicosecond time scales, driven by carrier-carrier scattering processes. As a reverse Auger recombination process, this model has been previously applied to account for the CM effect in bulk silicon ${ }^{45}$ and low-dimensional nanomaterials including QDs, ${ }^{12,46,49}$ carbon nanotubes, ${ }^{50}$ and $2 \mathrm{D}$ materials. ${ }^{47}$ Within the II framework, the CM efficiency is directly governed by the kinetic competition between II itself and hot carrier cooling. To evaluate the observed CM efficiency and compare the impact ionization rate to the cooling rate, we conduct a further data analysis following a phenomenological model proposed by Beard and colleagues. ${ }^{51}$ In the model, the detailed band structure of the material is neglected, and the $\mathrm{CM}$ efficiency is determined by the II and the carrier cooling via phonon emission.

Comparing the model (shown as dash-dotted lines in Figure $3 b$ ) to the data, we find the CM efficiency in $2 \mathrm{H}-\mathrm{MoTe}_{2}$ is nearly unity up to $\sim 94 \%$ (see details for the fitting in SI). The CM onset is found to be $\sim 2.8 E_{\mathrm{g}}$. Given the high $\eta_{C M}$, we can readily conclude that the II rate dominates over the cooling process in $2 \mathrm{H}-\mathrm{MoTe}_{2}$. Here we estimate the energy loss rate via cooling by $\left(h v-E_{\mathrm{g}}\right) / \tau_{\text {cooling }}$ (with $\tau_{\text {cooling }} \sim 1.5$ ps as the cooling time following $1.55 \mathrm{eV}$ excitation) to be $0.43 \mathrm{eV} / \mathrm{ps}$. We further assume a linear proportionality between the cooling time and the excess energy of hot carriers. Under such an assumption and applying the rate competition model by Beard, ${ }^{51}$ the II rate is estimated to be nearly 1 order of magnitude higher than the cooling rate for the first CM step (e.g., $3.2 \mathrm{eV} / \mathrm{ps}$ for $3.1 \mathrm{eV}$ excitation) to ensure a high $\mathrm{CM}$ efficiency in our system. The previously reported CM onset energy in $\mathrm{MoTe}_{2}$ of $\sim 2 E_{\mathrm{g}}$ is lower than the $2.8 E_{\mathrm{g}}$ found here. ${ }^{21}$ This difference may be due to variations in sample thickness and thus electronic structure but will require further study (see an extended discussion in SI).

While the phenomenological analysis captures the essence of $\mathrm{CM}$, to fully unveil the efficient II and thus CM in $2 \mathrm{H}-\mathrm{MoTe}_{2}$ theoretical calculations (e.g., by tight-binding calculations $)^{3,12,46}$ explicitly on multilayer $2 \mathrm{H}-\mathrm{MoTe}_{2}$ are required. To guide future theoretical treatments in this system, we summarize some key observations from the experiments.

(1) CM onset. In our study, the first CM onset and the accompanying sharp rise of the photoconductivity in $2 \mathrm{H}$ $\mathrm{MoTe}_{2}$ is found to be in the range of $2.4-2.6 \mathrm{eV}$, corresponding to the optical transition at $\Gamma$ point. To shed further insight on the $\mathrm{CM}$ at the $\Gamma$ point, we discuss the band structure of $\mathrm{MoTe}_{2}$ based on previous theoretical calculation 
(a)

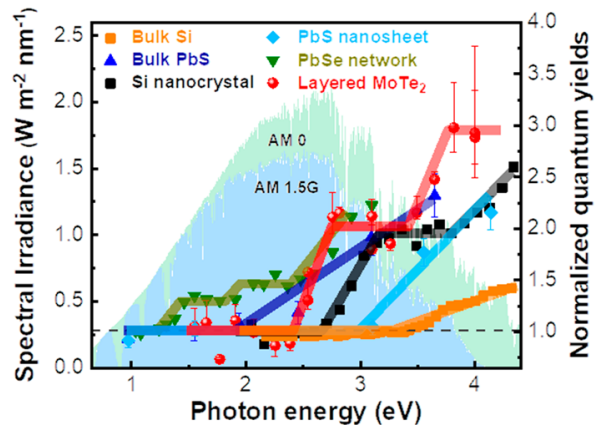

(b)

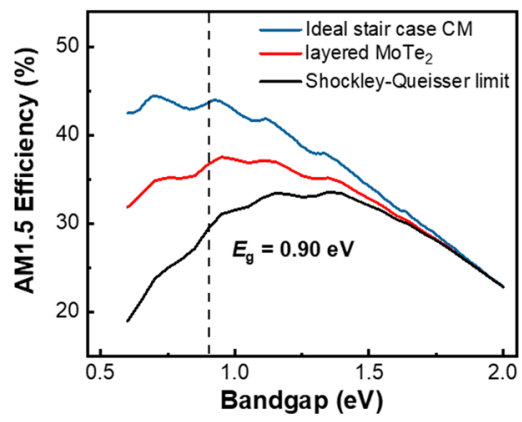

Figure 4. (a) $\mathrm{CM}$ efficiency comparison among $\mathrm{MoTe}_{2}$ and several other materials. (b) Simulated maximum power conversion efficiency of a $\mathrm{MoTe}_{2}$-based solar cell exposed to an AM1.5 solar spectrum and its comparison to the Shockley-Queisser limit and the ideal CM case.

(see a simplified scheme in Figure $3 \mathrm{c}) .^{52} \mathrm{MoTe}_{2}$ in excess of three layers exhibits an indirect bandgap involving the $K-\Lambda$ transition (with $E_{\mathrm{g}} 0.90 \mathrm{eV}$ ). For the first CM step, while the absorption takes place at $\Gamma$ point the $\mathrm{CM}$ involves the $K-\Lambda$ transition. Note that the valence band maximum (VBM) at $K$ and $\Gamma$ points are similar in energy. This results in a favorable "asymmetric" absorption condition, so that most of the excess energy is transferred to hot electrons. In line with our discussion here, Siebbeles et al. have also shown that asymmetric transitions are involved in optical transitions and responsible for the onset of $\mathrm{CM}$ effects in $\mathrm{PbS} \mathrm{QDs}$ and its bulk crystals. ${ }^{3}$ Overall, the indirect bandgap nature and the relatively close position of VBM at the $K$ and $\Gamma$ points make energy conservation readily achieved. Note that this characteristic band structure shares similarities with a fictitious band structure proposed by Queisser and his colleagues for optimal $\mathrm{CM}^{45}$ Finally, to fully understand why CM occurs efficiently at $\Gamma$ point insights into carrier cooling rate around $\Gamma$ point are also essential and call for further studies.

(2) Weak electron-phonon coupling in $\mathrm{MoTe}_{2}$. As we discussed, a large rate ratio between II and hot carrier cooling processes $k_{\mathrm{II}} / k_{\text {cool }}$ is required for efficient CM. Hence, efficient II and slow cooling dynamics favor CM. For the latter process, the weak coupling between the photogenerated hot carriers to phonon modes is desirable. For $\mathrm{MoTe}_{2}$ in both layered and bulk geometries, a few recent studies have reported an intrinsically weak carrier-phonon coupling. ${ }^{53-55}$ For instance, Makino et al. reported remarkably long-lived optical phonon modes, particularly the $A_{1 \mathrm{~g}}$ mode (with $\sim 20$ ps lifetime) in $\mathrm{MoTe}_{2}$, even in the presence of a large number of photoexcited carriers. ${ }^{55}$ This observation indicates very week coupling between photogenerated electrons and phonons; in conventional semiconductors (e.g., $\mathrm{GaN}, \mathrm{ZnO}$, and so forth), the electron-phonon coupling will lead to the coherent phonon dephasing on ultrafast, picosecond time scale. ${ }^{56,57}$ The unusual optical phonon dynamics and weak electron-phonon coupling strength could favor the CM by lifting the time constraint for II.

Implication. To illustrate the relevance of CM effect in $2 \mathrm{H}$ $\mathrm{MoTe}_{2}$ for optoelectronics, we compare the CM effect in $\mathrm{MoTe}_{2}$ to that in other extensively studied (e.g., $\mathrm{PbS}$ or PbSe systems) or technologically relevant materials (e.g., bulk Si or Si QDs), as shown in Figure 4a. While most of materials possess either only efficient CM (e.g., Si nanocrystals, ${ }^{43} \mathrm{PbS}$ nanosheets, ${ }^{20}$ and $\mathrm{PbSe}$ superlattice solids ${ }^{58}$ ) or high carrier mobility (e.g., bulk silicon ${ }^{59}$ ), $\mathrm{MoTe}_{2}$ uniquely combines both. This makes layered $\mathrm{MoTe}_{2}$ a fascinating material platform for both fundamental study of CM effect and further device integration. In this context, we further estimate the maximum power conversion efficiency (PCE) for our sample using the detailed balance model as shown in Figure $4 \mathrm{~b} .{ }^{60,61}$ In principle, the fast, sub-10 ps trapping process in $\mathrm{MoTe}_{2}$ imposes a challenge for its application in optoelectronics. Effective passivation schemes for the defects have been previously developed for other TMDC materials and should be applicable for $\mathrm{MoTe}_{2}$. For the calculation, we assume that defects can be effectively passivated so that all photogenerated carriers can be efficiently collected. With a quantized band gap of $0.90 \mathrm{eV}$, the maximum PCE can be reached to $36.8 \%$, increased from $29.5 \%$ without CM, with a substantial relative increase of $\sim 24.7 \%$, illustrating the relevance of $\mathrm{CM}$ effect in $\mathrm{MoTe}_{2}$ for optoelectronics.

\section{CONCLUSIONS}

In summary, we report that efficient photoconductivity or photocurrent multiplication occurs in few-layer $2 \mathrm{H}-\mathrm{MoTe}_{2}$ as a direct consequence of an efficient steplike carrier multiplication with near unity quantum yield and extremely high carrier mobility in $\mathrm{MoTe}_{2}$. We demonstrate that free carriers, rather than excitons, are involved in the CM process via impact ionization. The photocurrent multiplication, in conjunction with its $\sim 1 \mathrm{eV}$ bandgap and easy device integration, thanks to the $2 \mathrm{D}$ geometry, makes $\mathrm{MoTe}_{2}$ a promising candidate for hotcarrier related optoelectronics.

\section{ASSOCIATED CONTENT}

\section{St Supporting Information}

The Supporting Information is available free of charge at https://pubs.acs.org/doi/10.1021/acs.nanolett.0c01693.

Estimating band gap energy $E_{\mathrm{g}}$ using Tauc plots, the origin for the fast decay in carrier dynamics for few-layer $\mathrm{MoTe}_{2}$, photoconductivity dynamics of $2 \mathrm{H}-\mathrm{MoTe}_{2}$ with $3.1 \mathrm{eV}$ photoexcitation energy, $\mathrm{THz}$ time-domain spectroscopy, fitting the frequency-resolved data by Drude Model, comparison of the normalized frequencyresolved $\mathrm{THz}$ conductivity for excitations of 1.55 and 3.1 $\mathrm{eV}$, fitting the frequency-resolved data by Drude-Smith Model, the normalized, pump energy-dependent OPTP dynamics, discussion on CM onset energy difference between our studies and previous studies (PDF) 


\section{AUTHOR INFORMATION}

\section{Corresponding Author}

Hai I. Wang - Max Planck Institute for Polymer Research, 55128 Mainz, Germany; @ orcid.org/0000-0003-0940-

3984; Email: wanghai@mpip-mainz.mpg.de

\section{Authors}

Wenhao Zheng - Max Planck Institute for Polymer Research, 55128 Mainz, Germany

Mischa Bonn - Max Planck Institute for Polymer Research, 55128 Mainz, Germany; 이이이.org/0000-0001-6851-8453

Complete contact information is available at:

https://pubs.acs.org/10.1021/acs.nanolett.0c01693

\section{Author Contributions}

H.I.W. designed and supervised the project. W.Z. carried out the experimental work as well as the data analysis. W.Z. and H.I.W. wrote the manuscript. All authors have commented on the manuscript and given approval to the final version of the manuscript.

\section{Notes}

The authors declare no competing financial interest.

\section{ACKNOWLEDGMENTS}

We thank Heng Zhang, Shuai Fu, Alexander Tries, Xiaoyu Jia, Keno Krewer, Pankaj Seliya, and Paniz Soltani for useful discussions. We acknowledge Ronald Ulbricht and Heejae Kim for constructive comments on the manuscript.

\section{REFERENCES}

(1) Shockley, W.; Queisser, H. J. Detailed Balance Limit of Efficiency of p-n Junction Solar Cells. J. Appl. Phys. 1961, 32 (3), $510-519$.

(2) Schaller, R. D.; Klimov, V. I. High Efficiency Carrier Multiplication in PbSe Nanocrystals: Implications for Solar Energy Conversion. Phys. Rev. Lett. 2004, 92 (18), 186601.

(3) Spoor, F. C. M.; Grimaldi, G.; Delerue, C.; Evers, W. H.; Crisp, R. W.; Geiregat, P.; Hens, Z.; Houtepen, A. J.; Siebbeles, L. D. A. Asymmetric Optical Transitions Determine the Onset of Carrier Multiplication in Lead Chalcogenide Quantum Confined and Bulk Crystals. ACS Nano 2018, 12 (5), 4796-4802.

(4) Ten Cate, S.; Sandeep, C. S.; Liu, Y.; Law, M.; Kinge, S.; Houtepen, A. J.; Schins, J. M.; Siebbeles, L. D. Generating Free Charges by Carrier Multiplication in Quantum Dots for Highly Efficient Photovoltaics. Acc. Chem. Res. 2015, 48 (2), 174-181.

(5) Sandeep, C. S.; Cate, S.; Schins, J. M.; Savenije, T. J.; Liu, Y.; Law, M.; Kinge, S.; Houtepen, A. J.; Siebbeles, L. D. A. High ChargeCarrier Mobility Enables Exploitation of Carrier Multiplication in Quantum-Dot Films. Nat. Commun. 2013, 4, 2360.

(6) Mcguire, J. A.; Joo, J.; Pietryga, J. M.; Schaller, R. D.; Klimov, V. I. New Aspects of Carrier Multiplication in Semiconductor Nanocrystals. Acc. Chem. Res. 2008, 41 (12), 1810-1819.

(7) Schaller, R. D.; Agranovich, V. M.; Klimov, V. I. High-Efficiency Carrier Multiplication through Direct Photogeneration of MultiExcitons via Virtual Single-Exciton States. Nat. Phys. 2005, 1 (3), 189-194.

(8) Trinh, M. T.; Houtepen, A. J.; Schins, J. M.; Hanrath, T.; Piris, J.; Knulst, W.; Goossens, A. P. L. M.; Siebbeles, L. D. A. Spite of Recent Doubts Carrier Multiplication Does Occur in PbSe Nanocrystals. Nano Lett. 2008, 8 (6), 1713-1718.

(9) Trinh, M. T.; Polak, L.; Schins, J. M.; Houtepen, A. J.; Vaxenburg, R.; Maikov, G. I.; Grinbom, G.; Midgett, A. G.; Luther, J. M.; Beard, M. C.; Nozik, A. J.; Bonn, M.; Lifshitz, E.; Siebbeles, L. D. A. Anomalous Independence of Multiple Exciton Generation on
Different Group IV-VI Quantum Dot Architectures. Nano Lett. 2011, 11 (4), 1623-1629.

(10) Beard, M. C.; Knutsen, K. P.; Yu, P.; Luther, J. M.; Song, Q.; Metzger, W. L.; Ellingson, R. J.; Nozik, A. J. Multiple Exciton Generation in Colloidal Silicon Nanocrystals. Nano Lett. 2007, 7 (8), $2506-2512$.

(11) Beard, M. C.; Midgett, A. G.; Law, M.; Semonin, O. E.; Ellingson, R. J.; Nozik, A. J. Variations in the Quantum Efficiency of Multiple Exciton Generation for a Series of Chemically Treated PbSe Nanocrystal Films. Nano Lett. 2009, 9 (2), 836-845.

(12) Allan, G.; Delerue, C. Role of Impact Ionization in Multiple Exciton Generation in PbSe Nanocrystals. Phys. Rev. B: Condens. Matter Mater. Phys. 2006, 73 (20), 205423.

(13) Delerue, C.; Allan, G.; Pijpers, J. J. H.; Bonn, M. Carrier Multiplication in Bulk and Nanocrystalline Semiconductors: Mechanism, Efficiency, and Interest for Solar Cells. Phys. Rev. B: Condens. Matter Mater. Phys. 2010, 81 (12), 125306.

(14) Pijpers, J. J. H.; Ulbricht, R.; Tielrooij, K. J.; Osherov, A.; Golan, Y.; Delerue, C.; Allan, G.; Bonn, M. Assessment of CarrierMultiplication Efficiency in Bulk PbSe and PbS. Nat. Phys. 2009, 5 (11), 811-814

(15) Ben-Lulu, M.; Mocatta, D.; Bonn, M.; Banin, U.; Ruhman, S. On the Absence of Detectable Carrier Multiplication in a Transient Absorption Study of InAs/CdSe/ZnSe Core/Shell1/ Shell2 Quantum Dots. Nano Lett. 2008, 8 (4), 1207-1211.

(16) Nair, G.; Bawendi, M. G. Carrier Multiplication Yields of CdSe and CdTe Nanocrystals by Transient Photoluminescence Spectroscopy. Phys. Rev. B: Condens. Matter Mater. Phys. 2007, 76 (8), 081304.

(17) Nair, G.; Geyer, S. M.; Chang, L. Y.; Bawendi, M. G. Carrier Multiplication Yields in $\mathrm{PbS}$ and PbSe Nanocrystals Measured by Transient Photoluminescence. Phys. Rev. B: Condens. Matter Mater. Phys. 2008, 78 (12), 125325.

(18) Mak, K. F.; Lee, C.; Hone, J.; Shan, J.; Heinz, T. F. Atomically Thin $\mathrm{MoS}_{2}$ : a New Direct-Gap Semiconductor. Phys. Rev. Lett. 2010, 105 (13), 136805.

(19) Mak, K. F.; He, K.; Lee, C.; Lee, G. H.; Hone, J.; Heinz, T. F.; Shan, J. Tightly Bound Trions in Monolayer $\mathrm{MoS}_{2}$. Nat. Mater. 2013, 12 (3), 207-211.

(20) Aerts, M.; Bielewicz, T.; Klinke, C.; Grozema, F. C.; Houtepen, A. J.; Schins, J. M.; Siebbeles, L. D. A. Highly Efficient Carrier Multiplication in PbS Nanosheets. Nat. Commun. 2014, 5, 3789.

(21) Kim, J. H.; Bergren, M. R.; Park, J. C.; Adhikari, S.; Lorke, M.; Frauenheim, T.; Choe, D. H.; Kim, B.; Choi, H.; Gregorkiewicz, T. Lee, Y. H. Carrier Multiplication in Van Der Waals Layered Transition Metal Dichalcogenides. Nat. Commun. 2019, 10 (1), 5488.

(22) Tan, Y.; Luo, F.; Zhu, M.; Xu, X.; Ye, Y.; Li, B.; Wang, G.; Luo, W.; Zheng, X.; Wu, N.; Yu, Y.; Qin, S.; Zhang, X. A. Controllable 2Hto-1T' Phase Transition in Few-Layer MoTe ${ }_{2}$. Nanoscale 2018, 10 (42), 19964-19971.

(23) Kan, M.; Nam, H. G.; Lee, Y. H.; Sun, Q. Phase Stability and Raman Vibration of the Molybdenum Ditelluride $\left(\mathrm{MoTe}_{2}\right)$ Monolayer. Phys. Chem. Chem. Phys. 2015, 17 (22), 14866-14871.

(24) Empante, T. A.; Zhou, Y.; Klee, V.; Nguyen, A. E.; Lu, I. H.; Valentin, M. D.; Alvillar, S. A. N.; Preciado, E.; Berges, A. J.; Merida, C. S.; Gomez, M.; Bobek, S.; Isarraraz, M.; Reed, E. J.; Bartels, L. Chemical Vapor Deposition Growth of Few-Layer $\mathrm{MoTe}_{2}$ in the $2 \mathrm{H}$, 1T', and 1T Phases: Tunable Properties of $\mathrm{MoTe}_{2}$ Films. ACS Nano 2017, 11 (1), 900-905.

(25) Ruppert, C.; Aslan, O. B.; Heinz, T. F. Optical Properties and Band Gap of Single- and Few-Layer MoTe ${ }_{2}$ Crystals. Nano Lett. 2014, 14 (11), 6231-6236.

(26) Wilson, J. A.; Yoffe, A. D. The Transition Metal Dichalcogenides Discussion and Interpretation of the Observed Optical, Electrical and Structural Properties. Adv. Phys. 1969, 18 (73), 193-335.

(27) Fang, H.; Bechtel, H. A.; Plis, E.; Martin, M. C.; Krishna, S.; Yablonovitch, E.; Javey, A. Quantum of Optical Absorption in TwoDimensional Semiconductors. Proc. Natl. Acad. Sci. U. S. A. 2013, 110 (29), 11688-11691. 
(28) Yang, J.; Lu, T.; Myint, Y. W.; Pei, J.; Macdonald, D.; Zheng, J. C.; Lu, Y. Robust Excitons and Trions in Monolayer $\mathrm{MoTe}_{2}$. ACS Nano 2015, 9 (6), 6603-6609.

(29) Robert, C.; Picard, R.; Lagarde, D.; Wang, G.; Echeverry, J. P.; Cadiz, F.; Renucci, P.; Högele, A.; Amand, T.; Marie, X.; Gerber, I. C.; Urbaszek, B. Excitonic Properties of Semiconducting Monolayer and Bilayer $\mathrm{MoTe}_{2}$. Phys. Rev. B: Condens. Matter Mater. Phys. 2016, 94 (15), 155425.

(30) Han, B.; Robert, C.; Courtade, E.; Manca, M.; Shree, S.; Amand, T.; Renucci, P.; Taniguchi, T.; Watanabe, K.; Marie, X.; Golub, L. E.; Glazov, M. M.; Urbaszek, B. Exciton States in Monolayer $\mathrm{MoSe}_{2}$ and $\mathrm{MoTe}_{2}$ Probed by Upconversion Spectroscopy. Phys. Rev. X 2018, 8 (3), 031073.

(31) Sun, Y.; Zhang, J.; Ma, Z.; Chen, C.; Han, J.; Chen, F.; Luo, X.; Sun, Y.; Sheng, Z. The Zeeman Splitting of Bulk $2 \mathrm{H}-\mathrm{MoTe}_{2}$ Single Crystal in High Magnetic Field. Appl. Phys. Lett. 2017, 110 (10), 102102.

(32) Ulbricht, R.; Hendry, E.; Shan, J.; Heinz, T. F.; Bonn, M. Carrier Dynamics in Semiconductors Studied with Time-Resolved Terahertz Spectroscopy. Rev. Mod. Phys. 2011, 83 (2), 543-586.

(33) Dong, R.; Han, P.; Arora, H.; Ballabio, M.; Karakus, M.; Zhang, Z.; Shekhar, C.; Adler, P.; Petkov, P. S.; Erbe, A.; Mannsfeld, S. C. B.; Felser, C.; Heine, T.; Bonn, M.; Feng, X.; Canovas, E. High-Mobility Band-Like Charge Transport in a Semiconducting Two-Dimensional Metal-Organic Framework. Nat. Mater. 2018, 17 (11), 1027-1032.

(34) Tomadin, A.; Hornett, S. M.; Wang, H. I.; Alexeev, E. M.; Candini, A.; Coletti, C.; Turchinovich, D.; Kläui, M.; Bonn, M.; Koppens, F. H. L.; Hendry, E.; Polini, M.; Tielrooij, K. J. The Ultrafast Dynamics and Conductivity of Photoexcited Graphene at Different Fermi Energies. Sci. Adv. 2018, 4 (5), eaar5313.

(35) Xu, F.; Yu, C.; Tries, A.; Zhang, H.; Klaui, M.; Basse, K.; Hansen, M. R.; Bilbao, N.; Bonn, M.; Wang, H. I.; Mai, Y. Tunable Superstructures of Dendronized Graphene Nanoribbons in Liquid Phase. J. Am. Chem. Soc. 2019, 141 (28), 10972-10977.

(36) Ghimire, M. K.; Ji, H.; Gul, H. Z.; Yi, H.; Jiang, J.; Lim, S. C. Defect-Affected Photocurrent in $\mathrm{MoTe}_{2}$ FETs. ACS Appl. Mater. Interfaces 2019, 11 (10), 10068-10073.

(37) Grancini, G.; Maiuri, M.; Fazzi, D.; Petrozza, A.; Egelhaaf, H. J.; Brida, D.; Cerullo, G.; Lanzani, G. Hot Exciton Dissociation in Polymer Solar Cells. Nat. Mater. 2013, 12 (1), 29-33.

(38) Tries, A.; Osella, S.; Zhang, P.; Xu, F.; Ramanan, C.; Klaui, M.; Mai, Y.; Beljonne, D.; Wang, H. I. Experimental Observation of Strong Exciton Effects in Graphene Nanoribbons. Nano Lett. 2020, 20 (5), 2993-3002.

(39) Evers, W. H.; Schins, J. M.; Aerts, M.; Kulkarni, A.; Capiod, P.; Berthe, M.; Grandidier, B.; Delerue, C.; van der Zant, H. S. J.; van Overbeek, C.; Peters, J. L.; Vanmaekelbergh, D.; Siebbeles, L. D. A. High Charge Mobility in Two-Dimensional Percolative Networks of PbSe Quantum Dots Connected by Atomic Bonds. Nat. Commun. 2015, 6, 8195.

(40) Lan, X.; Chen, M.; Hudson, M. H.; Kamysbayev, V.; Wang, Y.; Guyot-Sionnest, P.; Talapin, D. V. Quantum Dot Solids Showing State-Resolved Band-Like Transport. Nat. Mater. 2020, 19 (3), 323329.

(41) Pradhan, N. P.; Rhodes, D.; Feng, X.; Xin, Y.; Memaran, S.; Moon, B.-H.; Terrones, H.; Terrones, M.; Balicas, L. Field-Effect Transistors Based on Few-Layered $\alpha-\mathrm{MoTe}_{2}$. ACS Nano 2014, 8 (6), $5911-5920$.

(42) Keum, D. H.; Cho, S.; Kim, J. H.; Choe, D. H.; Sung, H. J.; Kan, M.; Kang, H.; Hwang, J. Y.; Kim, S. W.; Yang, H.; Chang, K. J.; Lee, Y. H. Bandgap Opening in Few-Layered Monoclinic $\mathrm{MoTe}_{2}$. Nat. Phys. 2015, 11 (6), 482-486.

(43) Timmerman, D.; Valenta, J.; Dohnalova, K.; de Boer, W. D. A. M.; Gregorkiewicz, T. Step-Like Enhancement of Luminescence Quantum Yield of Silicon Nanocrystals. Nat. Nanotechnol. 2011, 6 (11), 710-713.

(44) Binks, D. J. Multiple Exciton Generation in Nanocrystal Quantum Dots-Controversy, Current Status and Future Prospects. Phys. Chem. Chem. Phys. 2011, 13 (28), 12693-12704.
(45) Werner, J. H.; Kolodinski, S.; Queisser, H. J. Novel Optimization Principles and Efficiency Limits for Semiconductor Solar Cells. Phys. Rev. Lett. 1994, 72 (24), 3851-3854.

(46) Franceschetti, A.; An, J. M.; Zunger, A. Impact Ionization Can Explain Carrier Multiplication in PbSe Quantum Dots. Nano Lett. 2006, 6 (10), 2191-2195.

(47) Michael, S.; Schneider, H. C. Impact Ionization Dynamics in Small Band-Gap Two-Dimensional Materials from a Coherent Phonon Mechanism. Phys. Rev. B: Condens. Matter Mater. Phys. 2019, 100 (3), 035431.

(48) Baer, R.; Rabani, E. Can Impact Excitation Explain Efficient Carrier Multiplication in Carbon Nanotube Photodiodes? Nano Lett. 2010, 10 (9), 3277-3282.

(49) Califano, M.; Zunger, A.; Franceschetti, A. Direct Carrier Multiplication due to Inverse Auger Scattering in CdSe Quantum Dots. Appl. Phys. Lett. 2004, 84 (13), 2409-2411.

(50) Gabor, N. M.; Zhong, Z.; Bosnick, K.; Park, J.; McEuen, P. L. Extremely Efficient Multiple Electron-Hole Pair Generation in Carbon Nanotube Photodiodes. Science 2009, 325, 1367-1371.

(51) Beard, M. C.; Midgett, A. G.; Hanna, M. C.; Luther, J. M.; Hughes, B. K.; Nozik, A. J. Comparing Multiple Exciton Generation in Quantum Dots to Impact Ionization in Bulk Semiconductors: Implications for Enhancement of Solar Energy Conversion. Nano Lett. 2010, 10 (8), 3019-3027.

(52) Sun, Y.; Wang, D.; Shuai, Z. Indirect-to-Direct Band Gap Crossover in Few-Layer Transition Metal Dichalcogenides: A Theoretical Prediction. J. Phys. Chem. C 2016, 120 (38), 2186621870.

(53) Li, T.; Zhang, Z.; Zheng, W.; Lv, Y.; Huang, F. A Possible High-Mobility Signal in Bulk $\mathrm{MoTe}_{2}$ : Temperature Independent Weak Phonon Decay. AIP Adv. 2016, 6 (11), 115207.

(54) Helmrich, S.; Schneider, R.; Achtstein, A. W; Arora, A.; Herzog, B.; de Vasconcellos, S. M.; Kolarczik, M.; Schops, O.; Bratschitsch, R.; Woggon, U.; Owschimikow, N. Exciton-Phonon Coupling in Monoand Bilayer $\mathrm{MoTe}_{2} .2 \mathrm{D}$ Mater. 2018, 5 (4), 045007.

(55) Makino, K.; Saito, Y.; Horii, S.; Fons, P.; Kolobov, A. V.; Ando, A.; Ueno, K.; Mondal, R.; Hase, M. Ultrafast Dynamics of ElectronPhonon Coupling in Transition-Metal Dichalcogenides. 2018, arXiv:1807.10879. Cond. Mat. Mtrl. Sci., https://arxiv.org/abs/ 1807.10879 (accessed Sep. 16 2019).

(56) Yee, K. J.; Lee, K. G.; Oh, E.; Kim, D. S.; Lim, Y. S. Coherent Optical Phonon Oscillations in Bulk GaN Excited by Far below the Band Gap Photons. Phys. Rev. Lett. 2002, 88 (10), 105501.

(57) Ishioka, K.; Petek, H.; Kaydashev, V. E.; Kaidashev, E. M.; Misochko, O. V. Coherent Optical Phonons of $\mathrm{ZnO}$ under Near Resonant Photoexcitation. J. Phys.: Condens. Matter 2010, 22 (46), 465803.

(58) Kulkarni, A.; Evers, W. H.; Tomic, S.; Beard, M. C.; Vanmaekelbergh, D.; Siebbeles, L. D. A. Efficient Steplike Carrier Multiplication in Percolative Networks of Epitaxially Connected PbSe Nanocrystals. ACS Nano 2018, 12 (1), 378-384.

(59) Wolf, M.; Brendel, R.; Werner, J. H.; Queisser, H. J. Solar Cell Efficiency and Carrier Multiplication in $\mathrm{Si}_{1-\mathrm{x}} \mathrm{Ge}_{\mathrm{x}}$ Alloys. J. Appl. Phys. 1998, 83 (8), 4213-4221.

(60) Hanna, M. C.; Nozik, A. J. Solar Conversion Efficiency of Photovoltaic and Photoelectrolysis Cells with Carrier Multiplication Absorbers. J. Appl. Phys. 2006, 100, 074510.

(61) Jensen, S. A.; Versluis, J.; Cánovas, E.; Pijpers, J. J. H.; Sellers, I. R; Bonn, M. Carrier Multiplication in Bulk Indium Nitride. Appl. Phys. Lett. 2012, 101 (22), 222113. 